\title{
Chemical Information of Chitosan-Based Complex Extracted from Coincidence Doppler Broadening Spectra
}

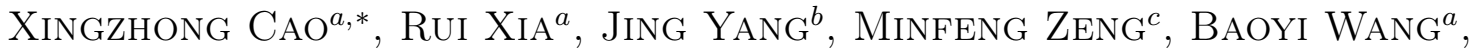 \\ RUNSHENG YU ${ }^{a}$ AND LONG $\mathrm{WEI}^{a}$ \\ ${ }^{a}$ Multi-discipline Research Center, Institute of High Energy Physics, Chinese Academy of Sciences, \\ No. 19 Yuquan Lu, Beijing 100049, China \\ ${ }^{b}$ Department of Applied Physics, Xi'an University of Sciences and Technology, Xi'an 710054, China \\ ${ }^{c}$ Zhejiang Key Laboratory of Alternative Technologies for Fine Chemicals Process, Shaoxing University, \\ Shaoxing 312000, China
}

\begin{abstract}
In recent years, coincidence Doppler broadening (CDB) of annihilation radiation measurement on polymeric system has been attracting special attention because of its unique elemental sensitivities in comparison to the traditional Doppler broadening measurement. In the present study, chitosan- $\mathrm{Fe}_{3} \mathrm{O}_{4}$ nanocomposite $\left(\mathrm{CS}-\mathrm{Fe}_{3} \mathrm{O}_{4}\right)$ and copper-chitosan $(\mathrm{Cu}-\mathrm{CS})$ complexes were prepared through a simple casting method from aqueous solution. The inclusion of $\mathrm{Fe}_{3} \mathrm{O}_{4}$ nanoparticles in the chitosan matrix is confirmed by X-ray diffraction. In order to obtain data about atomic composition of the annihilation site in the CS composites, CDB measurements were carried out. The normalized CDB ratio curve, which is related to the ratio between the momentum density distribution for the studied samples and pure CS, was displayed to illustrate the variation of ion cores of different elements present in the chitosan matrix. Our result confirms a contribution of active group available in chitosan molecules to the features in the high-momentum region of the CDB ratio curve. The findings show that the local chemical environment of the annihilation site in chitosan-based complexes could be estimated by such positron annihilation spectroscopic investigation.
\end{abstract}

DOI: 10.12693/APhysPolA.132.1535

PACS/topics: $78.70 . \mathrm{Bj}, 64.70 . \mathrm{km}, 82.30 . \mathrm{Gg}$

\section{Introduction}

In recent years, positron annihilation spectroscopy (PAS) considering its sensitivity and uniqueness at the atomic and molecular level has been attracting increasing attention in polymeric field [1-3]. Typically, free volume properties (e.g. size, density and size distribution), determined by ortho-positronium (o-Ps) lifetime and its corresponding intensity, have been widely used to explain the physicochemical or macroscopic properties correlated with sub-nanoscopic structural feature of polymeric material. However, little attention has been paid to the chemical surrounding of positron trapping sites in polymers, though it is of essential to the cognition of relevant positron annihilation characteristics $[4,5]$. Numerous studies indicate that the trapped positrons annihilate with the surrounding electrons, conveying useful information on the local electronic environment around the annihilate sites, and thus the high sensitivity of positrons to local chemical environment is well documented [6-8]. Significantly, the application of the CDB technique would enable the determination of the local environment, since it involves quantitative information on the detailed electron momentum distribution at positron annihilation site and thus can be further used to provide more chemical

*corresponding author; e-mail: caoxzh@ihep.ac.cn information about the local environment of the annihilation site [9-11]. In this work, in order to extract chemical information from chitosan-based complexes, coincidence Doppler broadening of the annihilation radiation measurement was performed at two different system, chitosan nanocomposite $\left(\mathrm{CS}-\mathrm{Fe}_{3} \mathrm{O}_{4}\right)$ and chitosan metal complex $(\mathrm{Cu}-\mathrm{CS})$, respectively.

\section{Experiment}

For preparing $\mathrm{CS}-\mathrm{Fe}_{3} \mathrm{O}_{4}$ nanocomposites, chitosan was dissolved in $2 \mathrm{wt} \%$ acetic acid solution at room temperature under magnetic stirring, and $\mathrm{Fe}_{3} \mathrm{O}_{4}$ nanopowder was then added to the solution which was again subjected to ultrasonic treatment for about $30 \mathrm{~min}$. The mixed solution was then cast on glass Petri dishes and dried at $310 \mathrm{~K}$ in vacuum for 2 days. The films contained 0.2 , $0.8,2,5,10 \mathrm{wt} \% \mathrm{Fe}_{3} \mathrm{O}_{4}$ and are referred to as $1-\mathrm{CSFe}$ - 5-CSFe, respectively. For preparing copper-chitosan (Cu-CS) complex, a certain volume amount of copper salt solution was slowly dropped into chitosan solution. The as-obtained blue solution was then transfered to glass Petri dish, and the air bubbles trapped in the viscous liquid were removed in vacuum. The finally formed films contained $2 \mathrm{~mL}$ volume of $\mathrm{CuCl}_{2}$ and $\mathrm{CuAc}_{2}$ solution; the films are referred to $\mathrm{CS}-\mathrm{Cl}^{-} \mathrm{Cu}$ and $\mathrm{CS}_{-}-\mathrm{ac}-\mathrm{Cu}$, respectively.

The structure of the $\mathrm{Fe}_{3} \mathrm{O}_{4}$ naoparticles and $\mathrm{CS}-\mathrm{Fe}_{3} \mathrm{O}_{4}$ nanocomposites was characterized by XRD (Rigaku 
XRD-3 Pgeneral with $\mathrm{Cu} \mathrm{K}_{\alpha}$ radiation). For CDB measurements, the positron source $\left({ }^{22} \mathrm{Na}, 16 \mu \mathrm{Ci}\right)$ sandwiched between two kapton foils of thickness $7 \mu \mathrm{m}$ was used for the CDB measurement at room temperature. Two identical HP-Ge detectors located at $180^{\circ}$ relative to each other were used to record the energies of two annihilation photons emitting from a single event, so as to reduce the background to a great extent. The central $511 \mathrm{keV}$ line is doppler-broadened due to the longitudinal component $P_{l}$ of the positron-electron momentum (Doppler shift $\Delta E=c P_{l} / 2, c$ is the speed of light) along the direction of the detector. Selection of coincidence events under the condition $2 m_{0} c^{2}-2.2 \mathrm{keV}<E_{1}+E_{2}<$ $2 m_{0} c^{2}+2.2 \mathrm{keV}$ was carried out: each $\mathrm{CDB}$ spectrum was recorded with the total counts equal to $1.2 \times 10^{7}$ in the coincidence $512 \times 512$-channel matrix. The data were analyzed in the form of ratio curve; each CDB spectrum was normalized to unit area and then re-normalized to the numeral data obtained for the reference sample to get the ratio curve.

\section{Results and discussion}

Figure 1a shows the XRD pattern for $\mathrm{Fe}_{3} \mathrm{O}_{4}$ nanoparticles and $\mathrm{CS}_{-}-\mathrm{Fe}_{3} \mathrm{O}_{4}$ nanocomposite films. The characteristic diffraction peaks for pure CS in the $2 \theta$ range of approximately 18 and $22 \div 23^{\circ}$ are observed [12]. On incorporation of $\mathrm{Fe}_{3} \mathrm{O}_{4}$, these peaks become broader indicating the disruption in the original structure of pure CS due to a reduction in intermolecular hydrogen bonding. On higher loading of filler, characteristic diffraction peaks corresponding to pure $\mathrm{Fe}_{3} \mathrm{O}_{4}$ are observed, thus giving a clear indication of the presence of $\mathrm{Fe}_{3} \mathrm{O}_{4}$ aggregates in the chitosan matrix.
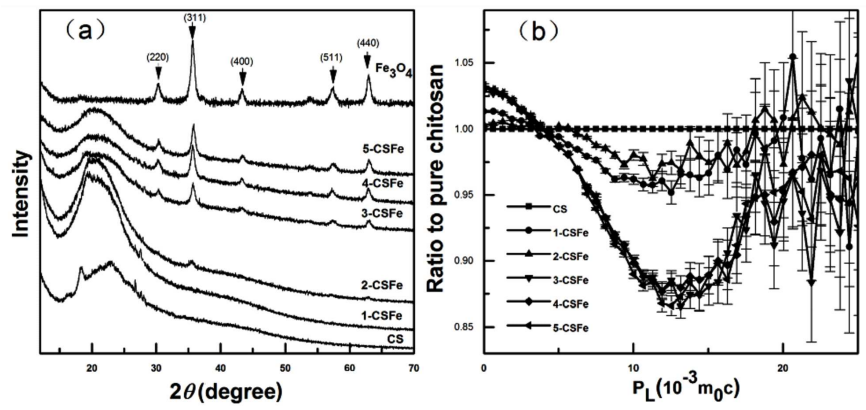

Fig. 1. (a) XRD patterns of $\mathrm{Fe}_{3} \mathrm{O}_{4}$ nanoparticles, pure chitosan, and $\mathrm{CS}-\mathrm{Fe}_{3} \mathrm{O}_{4}$ nanocomposite ( $n$-CSFe); (b) The CDB ratio curves of $\mathrm{CS}_{-} \mathrm{Fe}_{3} \mathrm{O}_{4}$ nanocomposites with respect to pure CS.

To obtain data related to elemental specificity of the atoms involved in the annihilation site in the nanocomposites, CDB measurements were carried out. To highlight the difference between the two CDB spectra, the constructed ratio curves are plotted in Figure 1b, which represent the normalized ratio for a certain spectral function of the momentum density distribution for $\mathrm{CS}-\mathrm{Fe}_{3} \mathrm{O}_{4}$ to pure CS. The horizontal axis expresses the momentum value of the annihilating positron-electron pairs in units of $10^{-3} m_{0} c$. These ratio curves show an evident variation in momentum range beyond $5 \times 10^{-3} m_{0} c$, for which semi-core electrons contribute mainly through non-Ps or free positron annihilation. Notably, the figure shows continuously reduction in the high momentum region $\left(5 \times 10^{-3} \div 20 \times 10^{-3} m_{0} c\right)$ over the range of loading from 0.2 to $2 \%$, whereas such reduction keeps unchanged on higher $\mathrm{Fe}_{3} \mathrm{O}_{4}$ loading (5\%). Since the chitosan molecule is consist of carbon, hydrogen, oxygen and nitrogen atoms, the above mentioned high-momentum region obtained for CS film is supposed to be proportional to the sum of contribution from carbon, nitrogen and oxygen. Thus the obvious reduction observed in this momentum range for as-obtained CDB ratio curve suggests a reduced contribution of positron annihilation with the semi-core electron states in chitosan nanocomposite. Since the semi-core electron of a specific element presented in the sample is not significantly influenced by its physical or chemical state, the elemental specificity or local chemical information could be obtained from the parameters of the CDB ratio curve. Theoretically, annihilation with $\mathrm{Fe}_{3} \mathrm{O}_{4}$ in nanocomposite would increase the ratio value of curve, since the $3 \mathrm{~d}$ orbital electrons for transition metal have a large contribution in high-momentum region. However, the reverse on the dropping trend is observed, implying that such a variation is mainly caused by the change of chemical environment for positron annihiltion. A similar type of change in the CDB ratio curve on incorporation of $\mathrm{NiO}$ as dopant in chitosan has also been reported [12]. This clearly indicates that most probable free positron annihilation site in $\mathrm{CS}-\mathrm{Fe}_{3} \mathrm{O}_{4}$ is the bulk of chitosan matrix or it is the interphase region where free positrons mostly annihilate with $\mathrm{H}$ and $\mathrm{C}$ atoms having high contribution in the lower momentum region, and the decrease in the area under the ratio curves is the result of the reduction in contribution from $\mathrm{N}$ and $\mathrm{O}$ element in polar $-\mathrm{NH}_{2}$ and $-\mathrm{OH}$ groups of chitosan molecules. In addition, the growing tendency of CDB ratio for all the samples in momentum region from $15 \times 10^{-3}$ to $20 \times 10^{-3} m_{0} c$ indicates the increase in contribution from semi-core electrons with higher momentum. This implies that the most probable annihilation site is $\mathrm{Fe}_{3} \mathrm{O}_{4}$ aggregates where free positrons annihilate with $3 \mathrm{~d}$ orbital electrons of transition metal having higher contribution in this momentum region.

For more qualitative discussion, the CDB spectra of standard $\mathrm{Cu}$ metal and $\mathrm{Cu}-\mathrm{CS}$ complexes (CS-ac-Cu, CScl-Cu) with respect to that of pure CS were also measured. Figure 2a shows that there is a peak around $20 \times 10^{-3} m_{0} c$ on the ratio curve of standard $\mathrm{Cu}$ metal. The result shows that the positrons annihilate with higher probability on the $3 \mathrm{~d}$ orbital electrons for transition metal in this momentum range. Figure $2 \mathrm{~b}$ shows the ratio curves of $\mathrm{CDB}$ spectra for the $\mathrm{CS}-\mathrm{ac}-\mathrm{Cu}$ complexes with respect to pure CS. For the sample doped with metallic $\mathrm{Cu}$ ions under its acetate form, the shape 


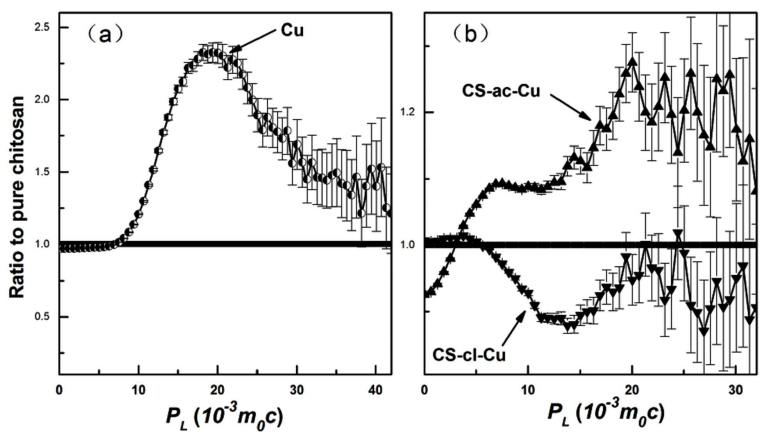

Fig. 2. $\mathrm{CDB}$ ratio curves of pure $\mathrm{Cu}(\mathrm{a}), \mathrm{CS}-\mathrm{Cl}-\mathrm{Cu}$ and $\mathrm{CS}-\mathrm{ac}-\mathrm{Cu}(\mathrm{b})$ with respect to pure CS.

of the ratio curve is very similar to that of the pure $\mathrm{Cu}$ curve. The peak position around $20 \times 10^{-3} m_{0} c$ is in accordance with that of pure $\mathrm{Cu}$ thus indicating that the information of $\mathrm{Cu}$ element could be effectively extracted from that of normalized ratio curve. According to the relation $E=P_{l}^{2} / 2 m_{0}$, the momentum of the semi-core electrons participating in the annihilation process around $12 \times 10^{-3} \mathrm{mc}$ has been identified as nitrogen-specific and $15 \times 10^{-3} \mathrm{mc}$ as oxygen-specific, indicative of a considerable positrons trapping by the $2 s$ electron shells of $\mathrm{N}$ and O, respectively $[7,9]$. The finding clearly shows that the other core electrons participating in the annihilation process in this momentum range are semi-core electrons of $\mathrm{N}$ and $\mathrm{O}$ atoms from $-\mathrm{NH}_{2}$ and $-\mathrm{OH}$ groups of chitosan, thus giving a clear indication of increasing probability localization of positron around $\mathrm{N}-\mathrm{Cu}$ and $\mathrm{O}-\mathrm{Cu}$ bond in copper-chitosan coordination compound.

On the other hand, the nature of the CDB ratio curve for CS-cl-Cu was very different. As it has already been mentioned above, if there had been much more nitrogen and oxygen contribution, the ratio curve should have been downward throughout the high-momentum region $\left(12 \div 18 \times 10^{-3} m_{0} c\right)$. However, it is noted that this shape of ratio curve is very similar to that of $\mathrm{CS}-\mathrm{Fe}_{3} \mathrm{O}_{4}$ in Fig. 1b. Clearly, the finding shows that free positrons are not trapped at the polar $-\mathrm{NH}_{2}$ and $-\mathrm{OH}$ groups in this case, and mainly annihilate from the chitosan backbone enriched by $\mathrm{C}$ and $\mathrm{H}$ atoms. The result is consistent with our previous research on CS-cl-Pd system, evidencing that additional negatively charged chloride ions existing in the surrounding of positively charged metallic ions and amino groups make great impact on local chemical environment for positron annihilation [4]. It may be mentioned that similar observation of preferential annihilation of positrons with electronegative chloride ions ligand has been discussed in detail in our previous paper On the other hand, the shape difference in the CDB curves around $20 \times 10^{-3} m_{0} c$ between CS-ac-Cu and CS-cl-Cu is small, indicating a similar metallic $\mathrm{Cu}$ contribution in them.

\section{Conclusion}

In summary, coincidence Doppler broadening of annihilation radiation has been measured for pure CS, CS$\mathrm{Fe}_{3} \mathrm{O}_{4}$ and $\mathrm{Cu}-\mathrm{CS}$ complexes. The constructed CDB ratio curve was used to understand the chemical environment of the annihilate site. The study shows that the application of the CDB technique enables the determination of the microscopic arrangement of atoms surrounding the annihilation site. Our experimental results confirm that the chemical information of annihilation site in polymer CS could be effectively extracted by such environmentdependent positron annihilation spectroscopic investigation. It inspires new perspectives of the application of positron annihilation technique for coordination chemistry.

\section{Acknowledgments}

This work was financially supported by National Nature Science Foundation of China (Grant No. 11575205, No. 11475197).

\section{References}

[1] S. Zekriardehani, S.A. Jabarin, D.R. Gidley, M.R. Coleman, Macromolecules. 50, 2845 (2017).

[2] S.K. Sharma, J. Prakash, J. Bahadur, K. Sudarshan, P. Maheshwari, S. Mazumder, P.K. Pujari, Phys. Chem. Chem. Phys. 16, 1399 (2014).

[3] Y.C. Jean, J.D. Van Horn, Hung Wei-Song, Lee Kuier-Rarn, Macromolecules 46, 7133 (2013).

[4] R. Xia, X.-Z. Cao, M.-Z. Gao, M.-F. Zeng, B.Y. Wang, L. Wei, Phys. Chem. Chem. Phys. 19, 3616 (2017).

[5] R.-W. Zhang, J. Robles, K. Jiwoong, Macromolecules 45, 2434 (2012).

[6] K. Sato, K. Ito, K. Hirata, R.S. Yu, Y. Kobayashi, Phys. Rev. B. 71, 012201 (2005).

[7] Y. Nagai, T. Nonaka, M. Hasegawa, Y. Kobayashi, C.-L. Wang, W. Zheng, C. Zhang, Phys. Rev. B. 60, 11863 (1999).

[8] X.-X. Hu, T. Koyanagi, Y. Katoh, B.D. Wirth, Phys. Rev. B. 95, 104103(2017).

[9] J. Dhar, S. Sil, A. Dey, P.P. Ray, D. Sanyal, J. Phys. Chem. Lett. 8, 1745-1751(2017).

[10] K. Sato, H. Murakami, K. Ito, K. Hirata, Y. Kobayashi, Macromolecules 42, 4853-4857 (2009).

[11] U. Myler, R.D. Goldberg, A.P. Knights, D.W. Lawther, P.J. Simpson, Appl. Phys. Lett. 69, 3333 (1996).

[12] S.K. Sharma, J. Bahadur, P.N. Patil, P. Maheshwari, S. Mukherjee, K. Sudarshan, S. Mazumder, P.K. Pujari, Chem. Phys. Chem. 14, 1055 (2013). 